\title{
FISSION YIELD MEASUREMENTS WITH THE ISOL METHOD
}

U. KÖSTER, G. AUBÖCK, U.C. BERGMANN, R. CATHERALL, J. CEDERKÄLL, L.M. FRAILE, S. FRANCHOO, H. FYNBO, U. GEORG, A. JOINET, O.C. JONSSON, J. LETTRY, K. PERÄJÄRVI, L. WEISSMAN ISOLDE, CERN, CH-1211 Genève 23

O. BAJEAT, R. BORCEA, C. BOURGEOIS, C. DONZAUD, M. DUCOURTIEUX, S. ESSABAA, D. GUILLEMAUD-MUELLER, F. HOSNI, F. IBRAHIM, C. LAU, H. LEFORT, J. OBERT, O. PERRU, J.C. POTIER, B. ROUSSIÈRE

Institut de Physique Nucléaire d'Orsay, F-91406 Orsay Cedex

\section{B. PFEIFFER}

Johannes-Gutenberg Universität, Institut für Kernchemie, D-55128 Mainz

E. FIORETTO, G. LHERSONNEAU, G. PRETE, L. STROE, L. TECCHIO Laboratori Nazionale di Legnaro, INFN, I-35020 Padova

C.AA. DIGET, H. JEPPESEN, K. RIISAGER

Aarhus Universitet, Institut for Fysik og Astronomi, DK-8000 Aarhus

H. GAUSEMEL, E. HAGEB $\varnothing$

University of Oslo, Department of Chemistry, N-0315 Oslo

D. RIDIKAS

CEA Saclay, DSM/DAPNIA/SPhN, F-91191 Gif-sur-Yvette

\footnotetext{
Radioactive ion beam intensities have been measured at ISOL (isotope separation on-line) facilities from many different targets, but only rarely these intensities are converted into production cross-sections. Here we discuss the method and possible problems in this conversion at the examples of $\mathrm{Kr}$ and $\mathrm{Xe}$ produced by 1.4 GeV-proton-induced fission of ${ }^{238} \mathrm{U}$ at ISOLDE and $\mathrm{Rb}$ and $\mathrm{Cs}$ produced by $\approx 10 \mathrm{MeV}$-neutron-induced fission of ${ }^{238} \mathrm{U}$ at PARRNe.
} 


\section{Introduction}

Fission product yields are important observables of the fission process. Mass yields have been measured for many fissioning systems at various excitation energies and it is well known that, with increasing excitation energy of the system, the valley of symmetry will be filled and the yields in the wings of the mass distribution (i.e. in far asymmetric fission) will be enhanced. However, isotopic yields are more difficult to obtain experimentally and are available for far fewer cases. An enlargement of our present knowledge of isotopic fission yields might not only help to improve our understanding of the fission process, but has also important practical applications. Fission is the most promising process for the production of very neutron-rich medium mass isotopes. Many different, already operating or projected, facilities use fission to produce intense beams of neutron-rich isotopes. They employ different projectiles at different energies:

- ${ }^{235} \mathrm{U}\left(\mathrm{n}_{\mathrm{th}}, \mathrm{f}\right):$ LOHENGRIN $^{1}$, OSIRIS $^{2}, M A F F^{3}$

- ${ }^{238} \mathrm{U}(\mathrm{p}, \mathrm{f})$ : ISOLDE $(1.0-1.4 \mathrm{GeV} \mathrm{p})^{4}$, LISOL $(30 \mathrm{MeV})^{5}$, JYFLIGISOL $(20+x \mathrm{MeV})^{6}$, HRIBF $(42 \mathrm{MeV})^{7}$

- $\mathrm{Ta} / \mathrm{W} / \mathrm{Hg}(\mathrm{p}, x \mathrm{n} ..) \rightarrow{ }^{238} \mathrm{U}(\mathrm{n}, \mathrm{f}): \operatorname{ISOLDE}(1.4 \mathrm{GeV} \mathrm{p})^{8}$, IRIS $(1.0$ $\mathrm{GeV})^{9}$, ISAC-2 $(0.5 \mathrm{GeV})^{10}, \quad$ EURISOL $(1.0 \mathrm{GeV})^{11}, \quad$ RIA $(0.9 \mathrm{GeV})^{12}$

- ${ }^{13} \mathrm{C}(\mathrm{p}, \mathrm{n}) \rightarrow{ }^{238} \mathrm{U}(\mathrm{n}, \mathrm{f}):$ SPES $(10-100 \mathrm{MeV} \mathrm{p})^{13}$

- ${ }^{12} \mathrm{C}(\mathrm{d}, \mathrm{n}) \rightarrow{ }^{238} \mathrm{U}(\mathrm{n}, \mathrm{f}):$ PARRNe $(26 \mathrm{MeV} \mathrm{d}){ }^{14}$, SPIRAL-II (40 $\mathrm{MeV})^{15}$

- ${ }^{9} \mathrm{Be}(\mathrm{d}, \mathrm{n}) \rightarrow{ }^{238} \mathrm{U}(\mathrm{n}, \mathrm{f}):$ PARRNe $(26 \mathrm{MeV} \mathrm{d})$

- $\mathrm{W}\left(\mathrm{e}^{-}, \gamma\right) \rightarrow{ }^{238} \mathrm{U}(\gamma, \mathrm{f}): \operatorname{DRIBS}\left(25 \mathrm{MeV} \mathrm{e}^{-}\right)^{16}, \operatorname{ALTO}(50 \mathrm{MeV})^{17}$

- ${ }^{1} \mathrm{H} /{ }^{6,7} \mathrm{Li} /{ }^{9} \mathrm{Be} /{ }^{208} \mathrm{~Pb}\left({ }^{238} \mathrm{U}, \mathrm{f}\right)$ : GSI-FRS (1 GeV/nucl. $)^{18}$, RIKEN $(0.35 \mathrm{GeV} / \text { nucl. })^{19}$, GSI-SIS200 (1.5 GeV/nucl. $)^{20}$, RIA $(0.4$ $\mathrm{GeV} /$ nucl. $)^{21}$

To select the optimum method for the production of the most neutron-rich nuclides we have to ask:

(1) How do the fission yields of a given element drop towards very neutron-rich isotopes?

(2) How depends the slope of the drop on the excitation energy of the fissioning system?

In the following we will discuss recent experiments contributing to these questions. 


\section{Experimental method}

We have to choose a separation and identification method which enables us to reach the most neutron-rich isotopes. The yields of these rare isotopes will be very low $\left(\leq 10^{-5}\right)$, hence a clear isotope identification can only be reached via a good $A$ and/or $Z$ separation. To study isotopes with a half-life of the order of $100 \mathrm{~ms}$ a rapid separation has to be performed online. For the study of fast-neutron-induced fission only the ISOL (isotope separation on-line) method provides sufficient luminosity. For ${ }^{238} \mathrm{U}(\mathrm{p}, \mathrm{f})$ either the ISOL method or in-flight separation in inverse kinematics (at the GSI-FRS) can be used.

The ISOL method uses thick targets, which are not necessarily "thick" for the incident beam (protons, neutrons, etc.), but "thick" for the reaction products. The latter will be fully stopped in the target matrix. The target is kept at a high temperature to assure a fast out-diffusion of the reaction products which will subsequently effuse towards an ion source where they are ionized to singly charged ions, extracted and accelerated to several ten $\mathrm{keV}$ and then mass-separated by deflection in a magnetic field. The $A$ separation is excellent (typically $A / \Delta A>1000$ ), but a $Z$ selectivity has to be introduced additionally.

\section{From "yields" to "yields"}

The mass-separated ion beam will be guided to a detection set-up to detect betas, beta-delayed gamma rays and/or beta-delayed neutrons. The event rate, corrected for the detection efficiency, the branching ratio and the transmission from the mass separator to the detection set-up gives the beam intensity. In the ISOL jargon the beam intensity normalized to the incident proton beam intensity is called "yield". This "ISOL yield" has to be corrected for the ionization efficiency and the release efficiency to obtain the (normalized) in-target production rate. The latter can be translated directly into the production cross-section when knowing the target thickness. Comparing with the total fission cross-section finally allows to deduce the "real" fission yields.

The ionization efficiency is element dependent, but to first order identical for isotopes of different mass. The release efficiency depends strongly on the half-life of the isotope in question. For a facility with dc primary beam and a known microstructure of the target, the release profile can be described by an analytical function. Fitting the latter to a release profile

measured for one or several isotopes allows to calculate the release efficiency 
for all isotopes of the same element. A detailed discussion of this procedure can be found in Refs. 14, 22.

For a facility like ISOLDE which uses a strongly bunched primary beam ( $2 \mu$ s bunch length, duty cycle $<2 \cdot 10^{-6}$ ) the release profile gets more complicated: shortly after the proton impact the target will experience a thermal spike (briefly accelerating the diffusion), radiation-induced dislocations in the target material will affect the diffusion, a bunched release of gas might affect the effusion, etc. Thus, for practical reasons we use an empiric 4 -parameter formula to fit the measured release profiles ${ }^{23,24}$. Depending on the used isotope, the measurement covers a dynamic range of some ms to many ten $\mathrm{s}$.

\section{Suitable elements}

To avoid a biasing of the results from a steep change in mass yield we should choose nuclides close to the peaks of the fission distribution where the mass yield behaves rather smoothly, i.e. ca. $86 \leq A \leq 104$ (corresponding to the elements $\mathrm{Br}-\mathrm{Zr}$ ) and $132 \leq A \leq 150$ (corresponding to Sn-Cs). Among these elements we should chose those which are released relatively quickly (to reach far out to short-lived isotopes) and which can be produced in a selective way (to assure a clear identification).

In the following we will discuss recent results from two measurements:

(1) $\mathrm{Kr}$ and $\mathrm{Xe}$ beams were produced at ISOLDE by $1.4 \mathrm{GeV}$ protoninduced fission in a ${ }^{238} \mathrm{UC}_{x} /$ graphite target ${ }^{24}$.

(2) At the PARRNe set-up at IPN Orsay fission was induced in a ${ }^{238} \mathrm{UC}_{x}$ /graphite target by neutrons created when stopping a $26 \mathrm{MeV}$ deuteron beam in a $3 \mathrm{~mm}$ thick graphite converter. The forward directed fast neutron spectrum was centered around $10 \mathrm{MeV}$ with a FWHM of $10 \mathrm{MeV}^{25}$. Surface-ionized $\mathrm{Rb}$ and Cs isotopes have been measured ${ }^{26}$.

\subsection{Noble gases}

Pure noble gas beams can be produced by isothermal chromatography, i.e. the target is connected via a water-cooled "distillation flask" to the ion source and only elements (or compounds) which are gaseous at room temperature will be able to reach the ion source. Noble gases have the advantage to give really cumulative cross-sections since the precursors $(\mathrm{Br}$, Se, ... ) are not released from the target and ion source unit, but decay 
inside. Moreover, the ionization efficiency can be monitored on-line by injecting a suitable support gas containing $\mathrm{Kr}$ and $\mathrm{Xe}$ via a calibrated leak and measuring the stable isotope currents. However, for very neutron-rich $\mathrm{Kr}$ and Xe isotopes (e.g. ${ }^{95} \mathrm{Kr}, 9$ mass units away from the stable ${ }^{86} \mathrm{Kr}$ and ${ }^{143} \mathrm{Xe}$, just 7 mass units away from the stable ${ }^{136} \mathrm{Xe}$ ) the branching ratios of the beta-delayed gamma rays are not well known or even no gamma ray energies are known at all. Hence these isotopes have to be identified and quantified by measuring the half-life of their beta decay with sufficient statistics.

Figures 1 and 2 show the fission yields of ${ }^{238} \mathrm{U}(\mathrm{p}, \mathrm{f})$ with $1.4 \mathrm{GeV}$ protons deduced from the ISOLDE yields in comparison to the independent yields of ${ }^{238} \mathrm{U}(\mathrm{p}, \mathrm{f})$ measured in inverse kinematics at $1.0 \mathrm{GeV}$ at GSI-FRS ${ }^{18}$, the cumulative yields calculated from the former, the independent yields of ${ }^{238} \mathrm{U}(\mathrm{n}, \mathrm{f})$ measured with a reactor neutron spectrum ${ }^{27}$, the yields calculated with MCNPX $^{29}$ (direct through proton-induced fission and total through proton- and neutron-induced fission) and the independent and cumulative recommended ${ }^{28}$ yields for a fast neutron spectrum.

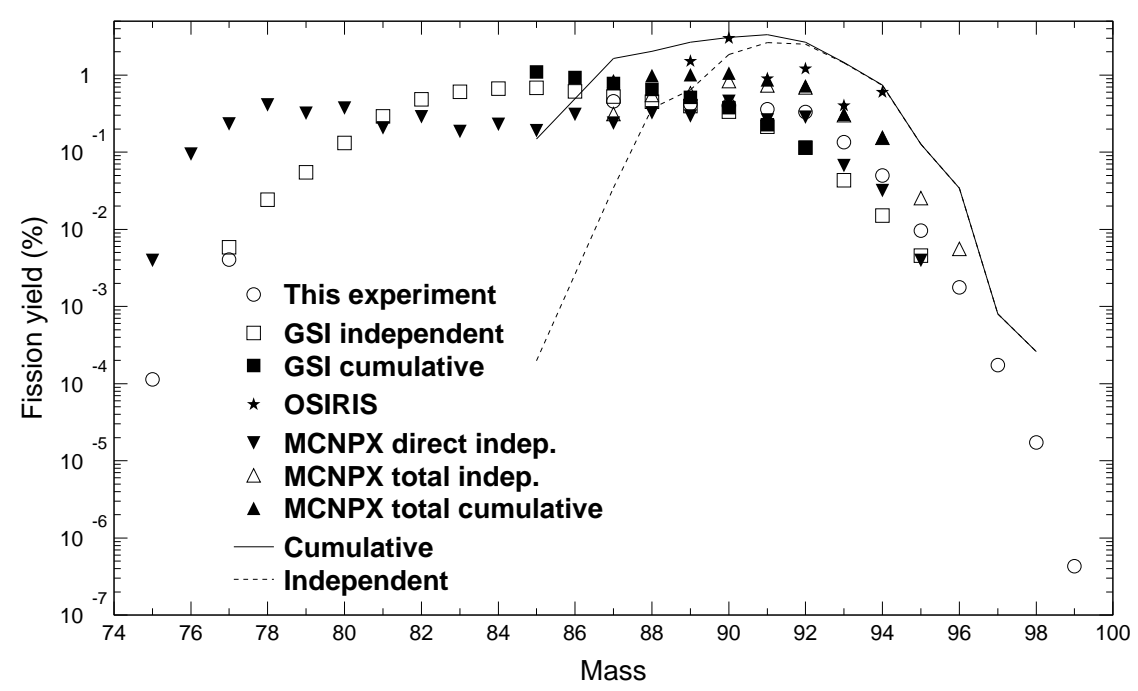

Figure 1. Measured and calculated fission yields of $\mathrm{Kr}$ isotopes. 


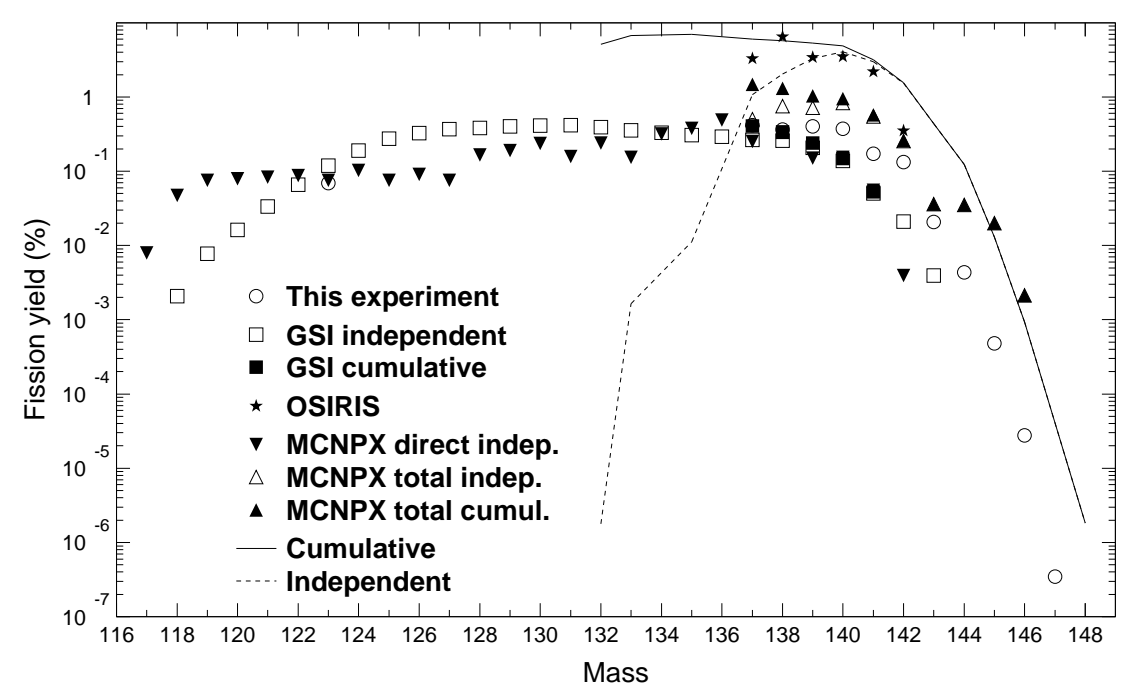

Figure 2. Measured and calculated fission yields of Xe isotopes.

\section{Contribution of secondary reactions}

Since we are using rather thick ISOL targets (typically $20 \mathrm{~cm}$ long) one has to be aware of a possible contribution of secondary reactions. The incident beam of high-energy protons can produce secondary particles (protons, neutrons, light charged particles, pions, etc.) which in turn might induce fission. To estimate the contribution of such secondary reactions we performed two types of calculations:

(1) With the Monte Carlo code from JAERI ${ }^{30}$ we simulated pencil beams of 2, 4 and $10 \mathrm{~mm}$ diameter and compared the relative production of fission fragments within the area of the incident beam to that in the laterally surrounding target area which is only intercepted by scattered protons and secondary particles. For a $4 \mathrm{~mm}$ diameter beam the outer target contributed at maximum $30 \%$ to the total production of slightly neutron-rich isotopes $\left({ }^{135-140} \mathrm{Xe}\right)$, but far less for the neutron-deficient and more neutron-rich nuclides.

(2) A second set of calculations was performed with the MCNPX code ${ }^{29}$ simulating the full UCx/graphite target geometry including the enclosing graphite and tantalum containers. It was found that the secondary reactions contribute $21 \%$ of all fission events. However, since this is mainly low-energy fission the effect close to the fission peaks is more pronounced and can reach up to a factor five enhance- 
ment. This calculation supports the explanation that the enhancement of the ISOLDE data compared to the GSI data is partly due to low-energy fission produced in secondary reactions and partly due to the difference of independent and cumulative cross-sections.

The size of the incident proton beam is actually measured slightly upstream of the target and then extrapolated with the nominal beam optics to the target position. There it is supposed to be elliptical with $\sigma_{\text {hor. }}=4 \mathrm{~mm}$ and $\sigma_{\text {vert. }}=2.5 \mathrm{~mm}$. An extremely conservative estimate of the uncertainty of the real beam size of $\pm 25 \%$ would, according to the MCNPX calculations translate into a change of the total fission rate by $-13 \% /+9 \%$. The ratio of direct to secondary reactions is not significantly affected.

\subsection{Alkalis}

Alkalis are another group of elements which is particularly favorable for the separation with the ISOL method. The heavier alkalis have very low ionization potentials ( $4.18 \mathrm{eV}$ for $\mathrm{Rb}$ and $3.89 \mathrm{eV}$ for $\mathrm{Cs}$ ) which allows to ionize them efficiently and selectively on the hot surface of a metal with high work function (e.g. W). Isobars with higher ionization potential are ionized far less efficiently. Beta-delayed gamma rays of alkali isotopes are known far out (up to ${ }^{102} \mathrm{Rb}, 15$ mass units away from stable ${ }^{87} \mathrm{Rb}$ and ${ }^{149} \mathrm{Cs}, 16$ mass units from stable ${ }^{133} \mathrm{Cs}$ ) and often also the branching ratios with sufficient precision. Moreover, the very neutron-rich isotopes of these elements have often high $P_{n}$ values which favors their identification via beta-delayed neutron detection. However, it is not easily possible to measure the ionization efficiency of alkalis on-line. Also, only a part of the precursors decays within the target, thereby populating the measured isotopes. Another part will leave the target and ion source unit as neutral atoms which will not reach the detection set-up. The released fraction will depend on the diffusion and effusion properties of the precursor elements, which in principle would need to be studied separately with a different ion source type where they can be ionized and detected after mass-separation. Hence, the measured yields are somewhere between independent and cumulative ones. For the very neutron-rich ones, the yield of the even more exotic precursor will be much smaller and can be safely neglected. Thus, for very neutron-rich isotopes the independent and cumulative yields will nearly coincide and are well represented by the measured yields. Since isotopic fission yields closer to stability can anyhow be measured more accurately with other methods the influence of the precursors is no principal drawback, but it remains a 
problem to find a suitable isotope for the overall normalization. This could either be a sufficiently neutron-rich isotope where the independent yield gets close to the cumulative yield (i.e. ${ }^{\geq 95} \mathrm{Rb}$ and ${ }^{\geq 146} \mathrm{Cs}$ ) or, even better, a shielded isotope, i.e. ${ }^{86} \mathrm{Rb}$ and ${ }^{136} \mathrm{Cs}$. Measurements of the latter from the same target and ion source unit are planned.

Figure 3 shows a preliminary evaluation for the fission yields measured at PARRNe in comparison to recommended ${ }^{28}$ values for the independent and cumulative yields with of $14 \mathrm{MeV}$-neutron-induced fission of ${ }^{238} \mathrm{U}$. The agreement looks satisfactory (in particular for the neutron-rich side), but the overall normalization factor for cesium was a factor two higher compared to rubidium. Since we do not expect such a big difference in the ionization efficiency this could indicate a systematic problem, possibly due to a very slow release component which remained unobserved.

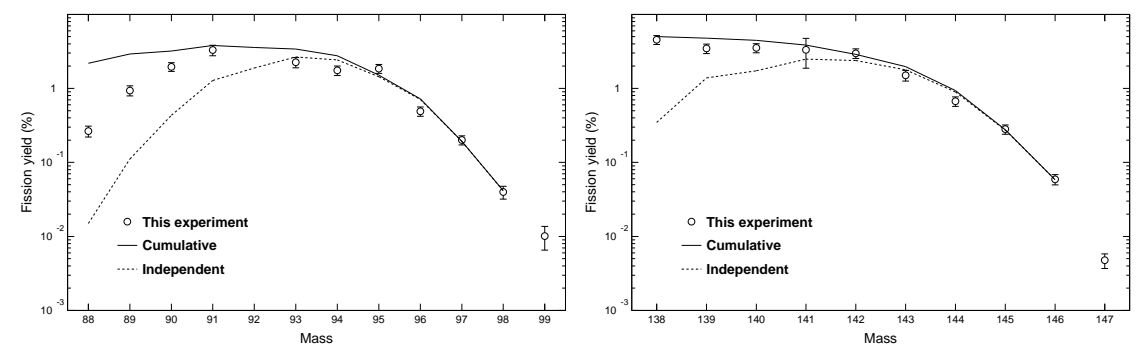

Figure 3. Measured and recommended fission yields of rubidium and cesium isotopes.

\section{Conclusion}

The transformation of ISOL beam intensities into production cross-sections requires a good understanding of the loss mechanisms in all steps from production to detection. This understanding is also required to reduce these losses and thus improve the intensity of existing radioactive ion beams. Hence a detailed study of this question can give a double benefit: more measured cross-sections and improved beams. The uncertainties of crosssections measured with the ISOL method will mostly remain dominated by systematic errors and rarely reach the accuracy of yields measured by in-flight facilities, but the much higher luminosity of ISOL facilities allows to reach far further out to exotic isotopes. Hence the driving force is the same as for all physics with exotic beams: A rough value measured far from stability may result in a much more stringent test of a model than a very precise measurement close to stability! 
More than 1000 measured ISOLDE yields are "waiting to be converted" into experimental cross-sections of spallation, fragmentation and fission. Collaborators interested in this analysis are very welcome!

\section{Acknowledgements}

Part of this work has been supported by the EU-RTD projects EURISOL (HPRI-CT-1999-50001) and TARGISOL (HPRI-CT-2001-50033).

\section{References}

1. P. Armbruster et al., Nucl. Instr. Meth. 139, 213 (1976).

2. B. Fogelberg et al., Nucl. Instr. Meth. B 70, 137 (1992).

3. T. von Egidy et al., Acta Phys. Slovaca 49, 107 (1999).

4. U. Georg et al., Nucl. Phys. A 701, 137c (2002).

5. K. Kruglov et al., Nucl. Phys. A 701, 145c (2002).

6. J. Äystö, Nucl. Phys. A 693, 477 (2001).

7. D. Stracener, Nucl. Instr. Meth. B 204, 42 (2003).

8. R. Catherall et al., Nucl. Instr. Meth. B 204, 235 (2003).

9. M. Portillo et al., Nucl. Instr. Meth. B 194, 193 (2002).

10. http://www.triumf.ca/isac/isac_home.html.

11. http://www.ganil.fr/eurisol.

12. B. Sherrill, Nucl. Instr. Meth. B 204, 765 (2003).

13. A. Andrighetto et al., Nucl. Instr. Meth. B 204, 267 (2003).

14. B. Roussière et al., Nucl. Instr. Meth. B 194, 151 (2002).

15. http://www.ganil.fr/research/developments/spiral2/index.html.

16. Y. Oganessian et al., Nucl. Phys. A 701, 87c (2002).

17. S. Essabaa et al., Nucl. Instr. Meth. B 204, 780 (2003).

18. M. Bernas et al., Nucl. Phys. A 725, 213 (2003).

19. T. Motobayashi, Nucl. Instr. Meth. B 204, 736 (2003).

20. H. Geissel et al., Nucl. Instr. Meth. B 204, 71 (2003).

21. J. Nolen et al., Nucl. Instr. Meth. B 204, 293 (2003).

22. C. Lau et al., to be published.

23. J. Lettry et al., Nucl. Instr. Meth. B 126, 130 (1997).

24. U. Bergmann et al., Nucl. Instr. Meth. B 204, 220 (2003).

25. S. Ménard et al., Phys. Rev. ST Accel. Beams 2, 033501 (1999).

26. C. Lau et al., Nucl. Instr. Meth. B 204, 257 (2003).

27. G. Rudstam et al., in Proc. of the Int. Conf. on Nuclear Data for Science and Technology, ed. by J. Dickens (American Nuclear Society, La Grange Park, Il, 1994), pp. 977-980.

28. T. England and B. Rider, Report LA-UR-94-106, ENDF-349, Los Alamos National Laboratory, USA (1994).

29. L. Waters, MCNPX code, http://mcnpx.lanl.gov/.

30. H. Takada et al., Report JAERI-Data/Code 98-005, Japan Atomic Energy Research Institute (1998). 